\title{
THE SCHWARZIAN DERIVATIVE AND QUASICONFORMAL REFLECTIONS ON $S^{n}$
}

\author{
Martin Chuaqui \\ University of Pennsylvania, Department of Mathematics \\ David Rittenhouse Laboratory, 209 South 33rd Street, Philadelphia, PA 19104-6395, U.S.A.
}

\begin{abstract}
It is well known that the Schwarzian derivative of an analytic map defined in a domain in the plane is closely related to global univalence and quasiconformal extension. Osgood and Stowe have recently found a generalization of the Schwarzian derivative for conformal local diffeomorphisms between Riemannian manifolds in arbitrary dimension. They establish a univalence criterion for such maps when the target is the sphere $S^{n}$. The condition is expressed as an inequality involving the norm of the generalized Schwarzian and quantities that depend on the geometry of the domain manifold. From this result it is possible to recover many injectivity criteria in the unit disc, including two classical conditions of Nehari. In connection with this work, we employ in this paper the techniques developed by C. Epstein to construct quasiconformal reflections in $S^{n}$ via hypersurfaces in hyperbolic $n+1$-space. Our main result shows that a strong form of the univalence criterion of Osgood and Stowe implies the existence of an orientation-reversing quasiconformal diffeomorphism of $S^{n}$ which fixes pointwise the boundary of the image of the map.
\end{abstract}

\section{Introduction}

In complex analysis, the Schwarzian derivative has played a salient role as a means of characterizing sufficient conditions for univalence and quasiconformal extension of analytic maps. These two phenomena are intimately related as a remarkable behavior often arises in the study of distortion theorems. Commonly, a stronger form of a given univalence criterion serves further as a condition that guarantees the existence of a quasiconformal extension to the entire plane. Gehring and Pommerenke have shown the following general result [Ge-Po]:

Let $\varrho \geq 0$ be a function defined in the unit disc $D$ such that the inequality

$$
|\{\psi, z\}| \leq \varrho(z) \quad \text { all } z \in D
$$

implies the univalence of the locally injective analytic map $\psi$. Here $\{\psi, z\}=$ $\left(\psi^{\prime \prime} / \psi^{\prime}\right)^{\prime}-\frac{1}{2}\left(\psi^{\prime \prime} / \psi^{\prime}\right)^{2}$ is the Schwarzian derivative of $\psi$. If

$$
|\{\psi, z\}| \leq t \varrho(z) \quad \text { all } z \in D
$$

for some $0 \leq t<1$, then $\psi$ is univalent and admits a $K$-quasiconformal extension to the plane. The constant $K$ depends only on $t$. They show that the result holds

1991 Mathematics Subject Classification: Primary 53A30; Secondary 30C65.

doi:10.5186/aasfm.1992.1720 
on quasidiscs and also when $\{\psi, z\}$ is replaced by the quantity $\psi^{\prime \prime} / \psi^{\prime}$. We remind the reader that a quasidisc is a Jordan domain which is the image of the unit disc under some quasiconformal map of the plane. Equivalently, a quasidisc is a Jordan domain whose boundary is the fixed point set of an orientation-reversing quasiconformal reflection of the extended plane.

Classical examples of (2) are [Ah 1], [A-W] and more recently, Epstein, and Anderson and Hinkkanen have derived very general theorems of univalence and quasiconformal extension (see [Ep 1] and $[\mathrm{A}-\mathrm{H}]$ ).

In a recent paper Osgood and Stowe have introduced a notion of Schwarzian derivative for conformal mappings of Riemannian manifolds, which generalizes the classical operator in the plane [O-S 1]. In the subsequent paper [O-S 2], and using their new notion, the same authors have established a sufficient condition for a conformal local diffeomorphism $\psi$ of a Riemannian $n$-manifold $(M, g)$ to the standard sphere $S^{n}$ to be injective (Theorem 1.1).

The criterion in [O-S 2] is classical in spirit, in the sense that it is stated as an inequality on the (generalized) Schwarzian derivative similar to (1). It is, on the other hand, very general in that it presents a unified approach to a vast class of known criteria. Different versions of the theorem can be obtained just by changing the metric $g$ conformally. For instance, Osgood and Stowe derive as corollaries with $M=D$ and $g$ alternately the euclidean and hyperbolic metric, two classical criteria of Nehari. Some new and most of the known criteria were derived from Theorem 1.1 in [Ch 1]. In particular, Epstein's injectivity result in [Ep 1] could be recovered in this fashion.

The purpose of this paper is to show that a phenomenon analogous to the one established by Gehring and Pommerenke also holds for the theorem of Osgood and Stowe. By using Epstein's techniques for constructing reflections in hyperbolic $(n+1)$-space, we will show that a strengthened version of Theorem 1.1 guarantees, in addition to the univalence of $\psi$, the existence of an orientation-reversing quasiconformal diffeomorphism of $S^{n}$ which fixes pointwise the boundary of the image $\psi(M)$. We shall follow Ahlfors in his definition of quasiconformality in higher dimensions.

The results of this paper were part of the author's dissertation, completed at Stanford in 1990 under the supervision of B. Osgood.

\section{Preliminaries}

In this section we will set up notation and present enough of the work in [O-S 1] so that we can state the injectivity result in [O-S 2].

Let $M$ be an $n$-dimensional Riemannian manifold with metric $g$. When $M=R^{n}$, we will denote by $g_{0}$ the euclidean metric and $g_{1}$ will stand for the standard metric on the sphere $S^{n}$. Given a conformal metric $\hat{g}=e^{2 \varphi} g$ on $M$, Osgood and Stowe define the Schwarzian tensor of $\hat{g}$ with respect to $g$ as the 
symmetric, trace free $(0,2)$-tensor

$$
B_{g}(\varphi)=\operatorname{Hess}(\varphi)-d \varphi \otimes d \varphi-\frac{1}{n}\left(\Delta \varphi-|\operatorname{grad} \varphi|^{2}\right) g
$$

where the metric dependent quantities on the right hand side are computed with respect to the metric $g$. When doing conformal changes of metric, the tensor $B_{g}(\varphi)$ appears as the term by which the trace free part of the Ricci tensors of $g$ and $e^{2 \varphi} g$ differ. We will make use of this later.

When $\psi$ is a conformal local diffeomorphism of $(M, g)$ to another Riemannian manifold $\left(N, g^{\prime}\right)$, then $\psi^{*}\left(g^{\prime}\right)=e^{2 \varphi} g$ with $\varphi=\log |D \psi|$. The Schwarzian derivative of $\psi$ is defined by

$$
S_{g}(\psi)=B_{g}(\varphi)
$$

For an analytic map $\psi$ in the plane with $g=g^{\prime}=g_{0}$, then $\varphi=\log \left|\psi^{\prime}\right|$ and computing in standard coordinates one gets

$$
S_{g}(\psi)=\left(\begin{array}{rr}
\operatorname{Re}\{\psi, z\} & -\operatorname{Im}\{\psi, z\} \\
-\operatorname{Im}\{\psi, z\} & -\operatorname{Re}\{\psi, z\}
\end{array}\right)
$$

where

$$
\{\psi, z\}=\left(\frac{\psi^{\prime \prime}}{\psi^{\prime}}\right)^{\prime}-\frac{1}{2}\left(\frac{\psi^{\prime \prime}}{\psi^{\prime}}\right)^{2}
$$

is the classical Schwarzian derivative.

On $M$, the conformal metric $\hat{g}=e^{2 \varphi} g$ is called Möbius with respect to $g$ if $B_{g}(\varphi)=0$, and so a conformal local diffeomorphism $\psi$ is said to be Möbius if $S_{g}(\psi)=0$. If $\varphi$ and $\sigma$ are smooth functions on $M$, then there is an important identity:

$$
B_{g}(\varphi+\sigma)=B_{g}(\varphi)+B_{\hat{g}}(\sigma)
$$

where $\hat{g}=e^{2 \varphi} g$. In a chain of conformal local diffeomorphisms $\psi_{1}:(M, g) \rightarrow$ $\left(N_{1}, g^{\prime}\right)$ and $\psi_{2}:\left(N_{1}, g^{\prime}\right) \rightarrow\left(N_{2}, g^{\prime \prime}\right)$, equation (1.2) can be formulated as

$$
S_{g}\left(\psi_{2} \circ \psi_{1}\right)=S_{g}\left(\psi_{1}\right)+\psi_{1}^{*}\left(S_{g^{\prime}}\left(\psi_{2}\right)\right) .
$$

This reduces to the classical formula for the Schwarzian derivative of a composition of analytic maps in the plane.

By $\left\|B_{g}(\varphi)\right\|$ we mean the norm of the Schwarzian tensor $B_{g}(\varphi)$ with respect to $g$, as a bilinear form on each tangent space, that is,

$$
\left\|B_{g}(\varphi)\right\|=\max \left\{\left|B_{g}(\varphi)(X, Y)\right|:|X|=|Y|=1\right\} .
$$

In cases, we will need to consider the norm of $B_{g}(\varphi)$ in a metric $\hat{g}=e^{2 \sigma} g$ conformal to $g$. Then

$$
\left\|B_{g}(\varphi)\right\|_{\hat{g}}=e^{-2 \sigma}\left\|B_{g}(\varphi)\right\| .
$$

With this, we now present the theorem in [O-S 2]. 
Theorem 1.1. Let $(M, g)$ be a Riemannian manifold of dimension $n \geq 2$ and $\psi:(M, g) \rightarrow\left(S^{n}, g_{1}\right)$ a conformal local diffeomorphism. Suppose that the scalar curvature of $M$ is bounded above by $n(n-1) K$ for some $K \in R$, and that any two points in $M$ can be joined by a geodesic of length $<\delta$ for some $0<\delta \leq \infty$. If

$$
\left\|S_{g}(\varphi)\right\| \leq \frac{2 \pi^{2}}{\delta^{2}}-\frac{1}{2} K
$$

then $\psi$ is injective.

With $M$ the unit disc in the plane and $g$ alternately the euclidean and hyperbolic metric, Osgood and Stowe derive from this theorem the classical criteria of Nehari:

$$
|\{\psi, z\}| \leq \frac{\pi^{2}}{2} \quad \text { or } \quad|\{\psi, z\}| \leq \frac{2}{\left(1-|z|^{2}\right)^{2}}, \quad \text { all }|z|<1
$$

implies that $\psi$ is univalent.

We point out that Theorem 1.1 can stated replacing $\left(S^{n}, g_{1}\right)$ by $\left(R^{n}, g_{0}\right)$ or $H^{n}$ with its metric of constant negative curvature. This follows from the transformation law (1.2) and the fact that both $g_{1}$ and the hyperbolic metric are Möbius with respect to the euclidean metric. Finally, let $\operatorname{scal}(g)$ be the scalar curvature of $g$. It it easy to verify that the proof given by Osgood and Stowe works equally well only assuming that at each point in $M$ the norm of the Schwarzian derivative of $\psi$ is bounded above by

$$
\frac{2 \pi^{2}}{\delta^{2}}-\frac{\operatorname{scal}(g)}{2 n(n-1)}
$$

\section{Reflections on $S^{n}$}

We think of $S^{n}$ as the ideal boundary of hyperbolic $(n+1)$-space $H^{n+1}$. Let $\Sigma$ be a complete hypersurface in $H^{n+1}$, with normal unit vector field $N$. At $p \in \Sigma$ we define $G_{+}(p), G_{-}(p) \in S^{n}$ by following the geodesic through $p$ normal to $\Sigma$ for infinite time, in the direction of $N$ and $-N$ respectively. Let $k_{1}, k_{2}, \ldots, k_{n}$ denote the principal curvatures of $\Sigma$. Epstein has shown that if $\left|k_{i}\right|<1$ for all $i$, then $\Sigma$ is embedded and $G_{+}$and $G_{-}$are diffeomorphisms onto open, disjoint simply-connected sets $\Omega_{+}$and $\Omega_{-}$in $S^{n}$. Furthermore, $\partial \Omega_{+}=\partial \Omega_{-}=\partial_{\infty} \Sigma$, the asymptotic boundary of $\Sigma$, and $S^{n}=\Omega_{+} \cup \Omega_{-} \cup \partial_{\infty} \Sigma$ [Ep 2]. This allows one to define the reflection $\Lambda=G_{-} \circ G_{+}^{-1}$, which maps $\Omega_{+}$onto $\Omega_{-}$and which extends to $\partial_{\infty} \Sigma$ as the identity.

For our purposes, $\Sigma$ will arise as the envelope of a family of horospheres $\{H(\theta, \varrho(\theta))\}$, parametrized by the points $\theta$ on a given domain $\Omega \subset S^{n}$ and a support function $\varrho$. The horosphere $H(\theta, \varrho)$ is tangent to $S^{n}$ at $\theta$ and is uniquely 
determined by $\varrho$, the (signed) distance between the horosphrere and a fixed origin $\vartheta$ in $H^{n+1}(\vartheta$ lies inside $H(\theta, \varrho)$ precisely when $\varrho<0)$.

According to [Ep 2],

$$
\Lambda(\theta)=\frac{|d \varrho|^{2}-1}{|d \varrho|^{2}+1} \theta+\frac{2 d \varrho}{|d \varrho|^{2}+1}
$$

where $d \varrho$ stands for the spherical gradient of $\varrho,|d \varrho|$ for its length in the spherical metric. It is then easy to see that

$$
d \varrho=\frac{\Lambda-(\Lambda \cdot \theta) \theta}{1-(\Lambda \cdot \theta)}
$$

where - is the euclidean inner product (points on $S^{n}$ are considered as being in $\left.R^{n+1}\right)$.

We want to express $d \varrho$ in terms of the stereographic coordinate $x=S(\theta)$ and the reflection

$$
w=S \circ \Lambda \circ S^{-1} \text {. }
$$

Let $X_{i}$ be the vector field on $S^{n}$ defined by $S_{*}\left(X_{i}\right)=\partial_{i}$. Then

$$
d \varrho\left(X_{i}\right)=d \varrho \cdot X_{i}=\frac{\Lambda \cdot X_{i}}{1-(\Lambda \cdot \theta)} .
$$

We now use the equations

$$
\Lambda \circ S^{-1}=S^{-1} \circ w=\left(1+|w|^{2}\right)^{-1}\left(2 w_{1}, \ldots, 2 w_{n},|w|^{2}-1\right)
$$

and

$$
X_{i}=2\left(1+|x|^{2}\right)^{-2}\left(-2 x_{1} x_{i}, \ldots, 1+|x|^{2}-2 x_{i}^{2}, \ldots,-2 x_{n} x_{i}, 2 x_{i}\right)
$$

to obtain

$$
d \varrho\left(X_{i}\right)=\frac{2 x_{i}}{1+|x|^{2}}+2 \frac{w_{i}-x_{i}}{|w-x|^{2}}
$$

We define

$$
f=\varrho \circ S^{-1}-\log \left(1+|x|^{2}\right)
$$

and so (2.3) yields

$$
\operatorname{grad} f=2 \frac{w-x}{|w-x|^{2}}
$$


or

$$
w=x+2 \frac{\operatorname{grad} f}{|\operatorname{grad} f|^{2}} .
$$

Here $\operatorname{grad} f$ stands for the euclidean gradient of $f$. We want to derive an expression for the quasiconformal distortion of $w$ in terms of $f$. We follow Ahlfors in his definition of quasiconformality [Ah 2]: let $D w$ be the differential of $w$, and consider the eigenvalues $\lambda_{1} \geq \lambda_{2} \geq \cdots \lambda_{n} \geq 0$ of the (positive) symmetric matrix $(D w)^{t}(D w)$. The map $w$ is said to be $K$-quasiconformal if $\lambda_{1} \lambda_{n}^{-1} \leq K^{2}$.

We therefore need to find upper and lower bounds for $|D w(y)|^{2}$, where $y \in R^{n}$ is a unit tangent vector at the point where the differential $D w$ is being considered. From (2.5),

$$
D w=I+2 D J(\operatorname{grad} f) \circ H(f)
$$

where $J(x)=x /|x|^{2}$ is the inversion in $R^{n}$. Its differential at the point $x$ is given by

$$
D J=|x|^{-4}\left(|x|^{2} I-2 Q(x)\right)
$$

here $Q(x)$ is the symmetric matrix with $i, j$-component $x_{i} x_{j}$. Note that $Q^{2}(x)=$ $|x|^{2} Q(x)$ and thus $D J$ is a conformal matrix such that $|D J|=|x|^{-2}$. Also, $H(f)$ stands for the Hessian of $f$, and in (2.6) $D J$ is evaluated at $x=\operatorname{grad} f$.

So we have

$$
D w(y)=y+2 D J(\operatorname{grad} f)(H(f)(y)) .
$$

We use \langle\rangle for the standard inner product in $R^{n}$ and compute:

$$
\begin{aligned}
|D w(y)|^{2} & =1+4|\operatorname{grad} f|^{-4}|H(f)(y)|^{2}+4\langle D J(\operatorname{grad} f)(H(f)(y)), y\rangle \\
& =1+4|\operatorname{grad} f|^{-4}|H(f)(y)|^{2}+4\langle H(f)(y), D J(\operatorname{grad} f)(y)\rangle .
\end{aligned}
$$

The Schwarzian tensor of $f$ with respect to the euclidean metric is defined so that the matrix $B(f)$ representing it, is given by

$$
B(f)=H(f)-Q(\operatorname{grad} f)-\alpha I,
$$

where $\alpha=\frac{1}{n}\left(\Delta f-|\operatorname{grad} f|^{2}\right)$. Thus

$$
\langle H(f)(y), y\rangle=\langle B(f)(y), y\rangle+\langle Q(\operatorname{grad} f)(y), y\rangle+\alpha
$$

and

$$
\begin{aligned}
|H(f)(y)|^{2}= & |B(f)(y)|^{2}+|Q(\operatorname{grad} f)(y)|^{2}+\alpha^{2}+2\langle B(f)(y), Q(\operatorname{grad} f)(y)\rangle \\
& +2 \alpha\langle B(f)(y), y\rangle+2 \alpha\langle Q(\operatorname{grad} f)(y), y\rangle
\end{aligned}
$$


and

$$
\begin{aligned}
\langle H(f)(y), Q(\operatorname{grad} f)(y)\rangle= & \langle B(f)(y), Q(\operatorname{grad} f)(y)\rangle \\
& +|\operatorname{grad} f|^{2}\langle Q(\operatorname{grad} f)(y), y\rangle+\alpha\langle Q(\operatorname{grad} f)(y), y\rangle .
\end{aligned}
$$

With this we obtain

$$
\begin{aligned}
|D w(y)|^{2}= & 1+4|\operatorname{grad} f|^{-4}|B(f)(y)|^{2}+4|\operatorname{grad} f|^{-4}|Q(\operatorname{grad} f)(y)|^{2} \\
& +4|\operatorname{grad} f|^{-4}\left(2 \alpha+|\operatorname{grad} f|^{2}\right)\langle B(f)(y), y\rangle+4 \alpha|\operatorname{grad} f|^{-2} \\
& +4 \alpha^{2}|\operatorname{grad} f|^{-4}-4|\operatorname{grad} f|^{-2}\langle Q(\operatorname{grad} f)(y), y\rangle \\
= & \left(1+2 \alpha|\operatorname{grad} f|^{-2}\right)^{2}+4|\operatorname{grad} f|^{-4}\left(|B(f)(y)|^{2}+\beta\langle B(f)(y), y\rangle\right) \\
& +4|\operatorname{grad} f|^{-4}\left(|Q(\operatorname{grad} f)(y)|^{2}-|\operatorname{grad} f|^{2}\langle Q(\operatorname{grad} f)(y), y\rangle\right),
\end{aligned}
$$

where

$$
\beta=2 \alpha+|\operatorname{grad} f|^{2}=\frac{2}{n} \Delta f+\left(\frac{n-2}{n}\right)|\operatorname{grad} f|^{2} .
$$

Using the fact that

$$
\begin{aligned}
|Q(\operatorname{grad} f)(y)|^{2} & =\langle Q(\operatorname{grad} f)(y), Q(\operatorname{grad} f)(y)\rangle \\
& =\left\langle Q^{2}(\operatorname{grad} f)(y), y\right\rangle=|\operatorname{grad} f|^{2}\langle Q(\operatorname{grad} f)(y), y\rangle,
\end{aligned}
$$

this finally yields:

Proposition 2.1. With the notation as before, the differential of the reflection $w$ satisfies

$$
|D w(y)|^{2}=4|\operatorname{grad} f|^{-4}|A(y)|^{2}
$$

where $A$ is the matrix given by

$$
A=\frac{1}{2} \beta I+B
$$

The scalar curvature of the metric $\hat{g}=e^{2 f} g_{0}$ is given by

$$
\operatorname{scal}(\hat{g})=-n(n-1) e^{-2 f} \beta,
$$

and the norm of the tensor $B_{g_{0}}(f)$ in the metric $\hat{g}$ is given by $e^{-2 f}\|B(f)\|_{g_{0}}$. Therefore, if

$$
\left\|B_{g_{0}}(f)\right\|_{\hat{g}} \leq \frac{t}{2} \frac{|\operatorname{scal}(\hat{g})|}{n(n-1)}
$$

for some $0 \leq t<1$, then

$$
(1-t)|\beta||\operatorname{grad} f|^{-2} \leq|D w(y)| \leq(1+t)|\beta||\operatorname{grad} f|^{-2} .
$$

Hence for $|\beta||\operatorname{grad} f| \neq 0$, the reflection $w$ will be $K$-quasiconformal with $K=$ $(1+t) /(1-t)$. 


\section{The main theorem}

Let $\psi:(M, g) \rightarrow\left(S^{n}, g_{1}\right)$ be a conformal local diffeomorphism. If

$$
\left\|S_{g}(\psi)\right\| \leq-\frac{t}{2} \frac{\operatorname{scal}(g)}{n(n-1)}
$$

for some $0 \leq t<1$, then $\psi$ is a global diffeomorphism and with $\phi=\psi^{-1}$, we can define the metric $g_{2}=e^{2} \varrho g_{1}=\phi^{*}(g)$ on $\Omega=\psi(M)$. We regard $\varrho$ as a support function defined on $\Omega$ and study the associated reflection $\Lambda$. The domain $\Omega$ will be simply-connected, a consequence of the following version of Theorem 1.1 [Ch 2]:

Theorem 3.1. Let $(M, g)$ be a complete Riemannian manifold of dimension $n \geq 2$ and $\psi:(M, g) \rightarrow\left(S^{n}, g_{1}\right)$ a conformal local diffeomorphism. If

$$
\left\|S_{g}(\psi)\right\| \leq-\frac{\operatorname{scal}(g)}{2 n(n-1)}
$$

then $M$ is simply-connected.

With the aid of (1.3) we will translate the inequality

$$
\left\|S_{g}(\psi)\right\| \leq-\frac{\operatorname{scal}(g)}{2 n(n-1)}
$$

to $\Omega$. Since $\phi^{*}(g)=e^{2 \varrho} g_{1}$, then

$$
B_{g_{1}}(\varrho)=S_{g_{1}}(\phi)=-\phi^{*}\left(S_{g}(\psi)\right)
$$

and therefore

$$
\left\|S_{g}(\psi)\right\|=\left\|B_{g_{1}}(\varrho)\right\|_{g_{2}} .
$$

Hence (3.1) is equivalent to

$$
\left\|B_{g_{1}}(\varrho)\right\|_{g_{2}} \leq-\frac{\operatorname{scal}\left(g_{2}\right)}{2 n(n-1)} .
$$

We use stereographic projection to pull back to the corresponding domain in euclidean space the conformal metric $g_{2}=e^{2 \varrho} g_{1}$ that is defined on $\psi(M)$. Let this new metric be written as $\hat{g}=e^{2 f} g_{0}$. Then $f$ and $\varrho$ are related to each other as in (2.4). Recall that when $\operatorname{scal}(\hat{g})|\operatorname{grad} f| \neq 0$, the quasiconformal distortion of the reflection $\Lambda$ is bounded by $(1+t) /(1-t)$, where

$$
t=\frac{2 n(n-1)}{|\operatorname{scal}(\hat{g})|}\left\|B_{g_{0}}(f)\right\|_{\hat{g}} .
$$


By construction, the metrics $\hat{g}$ and $g_{2}$ are isometric under the stereographic projection $S$, and furthermore we claim that

$$
\left\|B_{g_{0}}(f)\right\|_{\hat{g}}=\left\|B_{g_{1}}(\varrho)\right\|_{g_{2}} .
$$

This follows from the addition formula, as:

$$
0=B_{g_{0}}(f-f)=B_{g_{0}}(f)+B_{e^{2 f} g_{0}}(-f) .
$$

But

$$
B_{e^{2 f} g_{0}}(-f)=\left(S^{-1}\right)^{*}\left(B_{e^{2} \varrho g_{1}}(-\varrho)\right)=-\left(S^{-1}\right)^{*}\left(B_{g_{1}}(\varrho)\right) .
$$

Thus

$$
B_{g_{0}}(f)=\left(S^{-1}\right)^{*}\left(B_{g_{1}}(\varrho)\right)
$$

and our claim is established.

On the other hand, in [Ep 1] it is shown that the distortion of $\Lambda$ is given by

$$
\max _{i \neq j}\left|\left(\frac{1+k_{i}}{1-k_{i}}\right)\left(\frac{1-k_{j}}{1+k_{j}}\right)\right|,
$$

where $k_{1}, \ldots, k_{n}$ are the principal curvatures at the corresponding point $p=$ $G_{+}^{-1}(\theta)$ on $\Sigma$.

We want to conclude that $\left|k_{i}\right|<1$ for all $i$. For this we need to assume that $\operatorname{scal}\left(g_{2}\right)<0$, which will replace the apparently awkward condition $|\beta||\operatorname{grad} f| \neq 0$. We shall show that the assumption on $\operatorname{scal}\left(g_{2}\right)$ together with the inequality

$$
\left\|B_{g_{1}}(\varrho)\right\|_{g_{2}} \leq-\frac{t}{2} \frac{\operatorname{scal}\left(g_{2}\right)}{n(n-1)}
$$

for some $0 \leq t<1$, imply the sought estimate of the principal curvatures of $\Sigma$. Our first claim is that the metric $g_{2}$ is actually negatively curved. Indeed, since it is conformally flat, the Weyl tensor vanishes and hence the sectional curvatures are completely determined by the scalar curvature and the trace free part of the Ricci tensor. To be precise, for $X, Y$ orthonormal tangent vectors, the sectional curvature $K(X, Y)$ of $g_{2}$ is given by

$$
K(X, Y)=B_{g_{1}}(\varrho)(X, X)+B_{g_{1}}(\varrho)(Y, Y)+\frac{\operatorname{scal}\left(g_{2}\right)}{n(n-1)}
$$

(see [O-S 1], p. 24 for a classical decomposition of the curvature tensor we have used here). It follows that

$$
K(X, Y) \leq(1-t) \frac{\operatorname{scal}\left(g_{2}\right)}{n(n-1)}<0 .
$$


Let $\Sigma_{s}$ be the hypersurface parallel to $\Sigma$ at distance $s$. The hyperbolic metric $g_{s}$ on $\Sigma_{s}$, suitably normalized, converges as $s \rightarrow \infty$ to the metric $g_{2}$ on $\Omega$ [Ep 2]. The normalized sectional curvatures tend to $\left(k_{i} k_{j}-1\right)\left(1-k_{i}\right)^{-1}\left(1-k_{j}\right)^{-1}$, and therefore $\left(k_{i} k_{j}-1\right)\left(1-k_{i}\right)\left(1-k_{j}\right)<0$. Hence $k_{i} \neq 1$ for all $i$. Since the principal directions of $\Sigma$ and $\Sigma_{s}$ are mapped to each other under the parallel flow, this enables one to compute the differential of $\Lambda$. In particular, its determinant is given by

$$
-\prod_{i=i}^{n}\left(\frac{1+k_{i}}{1-k_{i}}\right)
$$

where $\left(1+k_{i}\right) /\left(1-k_{i}\right)$ is the eigenvalue of $d \Lambda$ corresponding to the principal direction $i$. Because $\Lambda$ reverses orientation, we conclude that

$$
\prod_{i=1}^{n}\left(1-k_{i}^{2}\right) \geq 0
$$

We claim that this inequality is strict. If this is not the case, then we must have $k_{i}=-1$ for some $i$ since we have already excluded the possibility $k_{i}=1$. Because $k_{i} \neq 1, D \Lambda$ does not have an infinite eigenvalue and therefore $|\operatorname{grad} f| \neq 0$ in (2.6). Hence the distortion is finite and we see from Epstein's formula that $k_{j}=-1$ for some $j \neq i$. This contradicts the fact that $\left(k_{i} k_{j}-1\right)\left(1-k_{i}\right)\left(1-k_{j}\right)<0$. This proves the claim, which now implies that $\#\left\{i:\left|k_{i}\right|>1\right\}$ is even. If this number is not zero, then say $\left|k_{1}\right|,\left|k_{2}\right|>1$. But then $\left(k_{1} k_{2}-1\right)\left(1-k_{1}\right)\left(1-k_{2}\right)>0$, again a contradiction. Therefore $\left|k_{i}\right|<1$ for all $i$, and so all the sectional curvatures of $\Sigma$ are negative.

Lemma 3.2. If $\varrho\left(\theta_{n}\right) \rightarrow \infty$ for any sequence $\left\{\theta_{n}\right\}$ in $\Omega$ converging (in the spherical metric) to a point in $\partial \Omega$, then $\Sigma$ is complete.

Proof. Suppose $\gamma(t)$ is a unit speed curve in $\Sigma$ defined on $[0,1)$ which cannot be extended continuously to $t=1$ in $\Sigma$. Then the curve $G_{+}(\gamma(t))$ in $\Omega$ will have to tend to $\partial \Omega$, hence $\varrho \rightarrow \infty$ along it. But then by construction of $\Sigma$ as the envelope of the horospheres $H(\theta, \varrho(\theta))$, we will have $\gamma(t)$ of infinite length, a contradiction.

We state now our main result.

Theorem 3.3. Let $(M, g)$ be a complete Riemannian $n$-manifold of negative scalar curvature, and let $\psi:(M, g) \rightarrow\left(S^{n}, g_{1}\right)$ be a conformal local diffeomorphism such that for some $t \in[0,1)$

$$
\left\|S_{g}(\psi)\right\| \leq-\frac{t}{2} \frac{\operatorname{scal}(g)}{n(n-1)} .
$$

Then $\psi$ is univalent and $M$ diffeomorphic to $R^{n}$. Furthermore, there exists a $(1+t) /(1-t)$-quasiconformal diffeomorphism $\Lambda$ of $S^{n}$ onto itself, which takes the topological hemisphere $\Omega=\psi(M)$ to $S^{n} \backslash \bar{\Omega}$ and which fixes $\partial \Omega$ pointwise. 
Proof. The univalence of $\psi$ follows from Theorem 1.1 and Theorem 3.1 implies that $M$, and thus $\Omega$, are simply-connected. As seen before, the condition on $S_{g}(\psi)$ corresponds to

$$
\left\|B_{g_{1}}(\varrho)\right\|_{g_{2}} \leq-\frac{t}{2} \frac{\operatorname{scal}\left(g_{2}\right)}{n(n-1)}
$$

and so $g_{2}$ and $g$ are negatively curved. The remaining conclusions follow from the Cartan-Hadamard theorem and the previous considerations on $\Lambda$.

We give the following application of this theorem. In [Ch 1] we derived a sufficient condition for univalence on an arbitrary simply-connectd domain $D_{1}$ in the plane. It states that if

$$
\left|l(z, z)-\frac{1}{6 \pi}\{\psi, z\}\right| \leq \frac{1}{3} K(z, \bar{z})
$$

for all $z \in D_{1}$, then $\psi$ is injective. The kernels $K$ and $l$ can be derived from the Green's function $h(z, \zeta)$ as follows:

$$
K(z, \bar{\zeta})=-\frac{2}{\pi} \frac{\partial^{2}}{\partial z \partial \bar{\zeta}} h(z, \zeta)
$$

and

$$
l(z, \zeta)=\frac{1}{\pi} \frac{1}{(z-\zeta)^{2}}+\frac{2}{\pi} \frac{\partial^{2}}{\partial z \partial \zeta} h(z, \zeta)
$$

$K$ is the Bergman kernel and the connection between these reproducing kernels and the theory of conformal mappings were extensively studied by Bergman and Schiffer [B-S]. The condition (3.7) appears as a corollary of Theorem 1.1 with $M=D_{1}$ and $g$ the Bergman metric $\pi K(z, \bar{z}) g_{0}$. We have therefore:

Corollary 3.4. Let $D_{1}$ be a simply-connected domain in the plane and let $\psi$ be analytic and locally injective in $D_{1}$. If

$$
\left|l(z, z)-\frac{1}{6 \pi}\{\psi, z\}\right| \leq \frac{t}{3} K(z, \bar{z})
$$

for all $z \in D_{1}$ and some $t, 0 \leq t<1$, then $\Omega=\psi\left(D_{1}\right)$ is a quasidisc.

Note that we cannot claim that $\psi$ admits a quasiconformal extension unless $D_{1}$ is itself a quasidisc.

Finally, the results presented here yield the following sufficient condition for a planar domain to be a quasidisc:

Corollary 3.5. Let $\Omega \subset R^{2}$ be a domain and let $g=e^{2 f} g_{0}$ be a complete metric of negative Gaussian curvature $k(g)$ on $\Omega$. If for some $t \in[0,1)$

$$
\left\|B_{g_{0}}(f)\right\|_{g} \leq-\frac{t}{2} k(g)
$$

then $\Omega$ is a quasidisc. 
The inequality (3.8) can be written more simply in the form

$$
\left|f_{z z}-f_{z}^{2}\right| \leq t f_{z \bar{z}}
$$

This theorem is implicit in the work of Epstein, but was never stated in this intrinsic form.

\section{References}

[Ah 1] AhLFors, L.V.: Sufficient conditions for quasiconformal extensions. - Discontinuous groups and Riemann surfaces, Ann. of Math. Stud. 79, Princeton University Press, 1974.

[Ah 2] Ahlfors, L.V.: Schwarzian derivative and cross-ratio in $R^{n}$. - In Complex Analysis: A Collection of Papers Dedicated to Albert Pfluger, Birkhäuser, Boston, 1989.

[Ah-W] Ahlfors, L.V., and G. Weill: A uniqueness theorem for Beltrami equations. - Proc. Amer. Math. Soc. 13, 1962, 975-978.

[A-H] Anderson, J., and A. Hinkkanen: Univalence criteria and quasiconformal extensions. - Trans. Amer. Math. Soc. 324, 1991, 823-842.

[B-S] Bergman, S., and M. Schiffer: Kernel functions and conformal mapping. - Compositio Math. 8, 1951, 205-249.

[Ca] Carne, K.: Schwarzian derivative for conformal maps. - J. Reine Angew. Math. 408, 1990, 10-33.

[Ch 1] Chuaqui, M.: A unified approach to univalence criteria in the unit disc and simplyconnected domains. - To appear.

[Ch 2] Chuaqui, M.: Ricci curvature and a criterion for simple-connectivity on the sphere. - To appear.

[Ch-O 1]Chuaqui, M., and B. Osgood: The Schwarzian derivative and conformally natural quasiconformal extensions in one, two and three dimensions. - Math. Ann. 292:2, 1992, 267-280.

[Ch-O 2]Chuaqui, M., and B. Osgood: Sharp distortion theorems associated with the Schwarzian derivative. - Proc. London Math. Soc. (to appear).

[Ep 1] Epstein, C.: The hyperbolic Gauss map and quasiconformal reflections. - J. Reine Angew. Math. 372, 1986, 96-135.

[Ep 2] Epstein, C.: Envelopes of horospheres and Weingarten surfaces in hyperbolic space. Preprint, 1984.

[Ge-Po] Gehring, F.W., and Ch. Pommerenke: On the Nehari criterion and quasicircles. Comment. Math. Helv. 59, 1984, 226-242.

[O-S 1] Osgood, B., and D. STowe: The Schwarzian derivative and conformal mapping of Riemannian manifolds. - Duke Math. J. (to appear).

[O-S 2] Osgood, B., and D. Stowe: A generalization of Nehari's univalence criterion. - Comment. Math. Helv. 65:2, 1990, 234-242.

[Po] Pommerenke, Ch.: On the Epstein univalence criterion. - Resultate Math. 10, 1986, 143-146. 